\title{
Multi-Agent Simulation of Ground Aircraft Operations at a Large Airport
}

$$
\begin{aligned}
& \text { 7-3-1 Hongo, Bunkyo-ku } \\
& \text { Tokyo 113-8656, Japan } \\
& +81-3-5841-6965
\end{aligned}
$$

Shun Nakamura, Kazuo Furuta, Taro Kanno

Dept. Systems Innovation, The University of Tokyo

\{nakamura, fuaura, kanno\}@sys.t.u-tokyo.ac.jp

\author{
Shigeki Yoshihara, Takamichi Mase \\ ANA Strategic Research Institute Co., Ltd. \\ 1-5-2 Higashi-shimbashi, Minato-ku \\ Tokyo, 105-7133, Japan \\ +81-3-6735-1464
}

\{s.yoshihara, ta.mase\}@ana.co.jp

\begin{abstract}
How to realize efficient ground aircraft operations at a large airport is a key issue to respond increasing air traffic demands. This study aims at establishing a systematic and concrete method for assessing not only ground facility designs but also operation schemes in terms of aircraft operations on airfields. A multi-agent simulation system was developed for ground aircraft operations based on the simulation model constructed through field visit and observation considering implicit action rules of field experts. An interactive design process of visualizing simulation results, showing them to field experts, getting their opinions, and considering the obtained feedbacks is very effective for brush-up of the simulation model or design plans. The simulation aims directly at assessing efficiency of operations plans, and finally at improving efficiency through interactive process with experts of proposal, simulation, and review.
\end{abstract}

Comparison of simulation results with the field data demonstrated that the simulation system can well predict the actual operation performance and that it is useful to comparatively assess design plans for ground aircraft operations. The method proposed was then applied to a case study to assess the operation plans expected after the scheduled extension of a runway and a terminal building at Tokyo International Airport (Haneda). As a result of simulation, several problems in the present plans could be pointed out, and the findings will be useful to revise them.

\section{Categories and Subject Descriptors}

I.6.5 [Simulation and Modeling]: Model Development Modeling methodologies; J.2 [Physical Sciences and Engineering]: Aerospace.

\section{General Terms}

Design.

Permission to make digital or hard copies of all or part of this work for personal or classroom use is granted without fee provided that copies are not made or distributed for profit or commercial advantage and that copies bear this notice and the full citation on the first page. To copy otherwise, or republish, to post on servers or to redistribute to lists, requires prior specific permission and/or a fee.

SIMUTools 2010 March 15-19, Torremolinos, Malaga, Spain.

Copyright 2010 ICST, ISBN 78-963-9799-87-5.

\section{Keywords}

Service Design, Ground Aircraft Operations, Multi-Agent Simulation, Human Modeling, Ethnography.

\section{INTRODUCTION}

Due to the increasing air traffic demands in recent years, congestion of air traffic not only in airspaces but also on airfields is coming to be a serious problem. Extension of runways and terminals are planned therefore at large airports worldwide to overcome this problem, but solutions just with hardware facilities do not necessarily work. Efficient management of aircraft operations on airfields is expected at the same time in air transportation services.

Previous works dealt with ground aircraft operations in preparation for extension of runway capacity [1], but hardware design often used to attract more interests of designers than operation schemes. A model for total airport performance analysis and a decision support system for performance assessment were developed [2], but this model does not consider microscopic processes of aircraft operations. As for ground aircraft operations at an airport, a time-dependent network assignment strategy was applied to aircraft taxiway operations [3], and discrete-event simulation to runway operations [4].

SIMMOD [5], developed by the US Federal Aviation Administration (FAA), will be the most widely used model for detailed and dynamic simulation of aircraft operations. It is based on a node-link network model of airfields and airspaces. SOMMOD is also discrete-event simulation, and it is assumed that the state of model changes at discrete points in time at which events occur. The event schedule is determined stochastically with event queues. Since SIMMOD is a one-dimensional model, which consists of multiple path segments of aircrafts, it does not check interference of aircrafts in different path segments in lateral directions. In addition, the modelers must predefine special nodes or areas to simulate properly congestion and staging of aircraft. This feature and the stochastic modeling framework can be limitations of SIMMOD for simulating local interference of aircraft precisely.

How to model human performance is another research issue. In aerospace industries, human performance modeling and simulation environments have been developed for design, visualization, and assessment of complex man-machine systems 
for cockpit design [6], but such technologies can hardly be applied directly to aircraft operations where interactions among more agents play crucial roles than in a cockpit. The simulation models developed for aircraft operations rarely contain human performance model explicitly. Other researchers studied arrival passenger flow with a queuing network system for efficient use of gates [7], but aircraft operations on taxiways were beyond the scope of these studies.

These previous studies did not consider microscopic interactions of aircraft like delays caused by inappropriate ordering or pattern of pushback operations in departure. Our interview to operation managers of an airline suggested that such interactions influence efficiency of aircraft operations considerably. In this study, we will try to construct a simulation model for ground aircraft operations from a microscopic view so that we can assess and design the whole system of hardware and humans performance considering dynamic and detailed operation processes.

The aim of this work is to propose a method of simulation for ground aircraft operations at a large airport considering the implicit rules extracted from field experts and to develop a simulation system that can be used for designing operation plans of aircraft on airfields. By comparing simulation results with the data obtained through field observation, the reality of simulator was evaluated. To demonstrate further usefulness of the proposed method, it was applied to assess the operation plans now under consideration after the scheduled extension of a runway and a terminal building at Tokyo International Airport.

\section{SIMULATION MODEL}

\subsection{Field Visit and Observation}

Since human expertise of field staff plays important roles in ground aircraft operations, we adopted an ethnographic approach, which is a study method of sociology to find out some implicit orders, rules, or norms behind human behavior through observation in the actual work environment [8,9]. We visited Tokyo International Airport several times and carried out field observation to examine the reality of work. The places visited include ground facilities, the operation control center of an airline, towing vehicles, and so on. In addition we interviewed field staff in various sections of the airline. The interviewees were operators of towing cars, pilots, and operation controllers. As a result, the flow of activities and the roles of staff were clarified. Documents on the layout and specs of ground facilities were separately obtained.

Movements of aircraft were video-recorded and radio communication between pilots and ground controllers was voicerecorded. A time period of standard operation conditions during daytime was chosen for the recording. The total recording time exceeded ten hours. From the video and voice data, the time required for arrival and that for departure were evaluated and taxiing routes instructed by ground controllers were identified. These data were then transcribed and used as a basis for acquiring the action rules that pilots and ground controllers use in their work. Pushback patterns used for each spot were recorded and tabulated with their application conditions. For example, normative pushback patterns are determined from the usage of runways to shorten the taxiing distance to the runways. It can, however, be modified due to local interference with some aircraft nearby already on the taxiway. Such rules reside in minds of field experts.

Preliminary results of simulation were shown to operation controllers, pilots, ex-pilots, and ex-controllers for qualitative check in the course of model development by prototyping. Obtained comments were fed back to modification of the simulation model, and this process was repeated a few times. Such an interactive approach was essential to consider tacit knowledge possessed by field experts.

\subsection{Overview}

An overview of the simulation model is illustrated in Figure 1, where thin black arrows represent service delivery and thick gray ones reference of information. Aircraft operation service consists primarily of three components: ground (air traffic) control service between ground controllers and aircraft, transportation service between aircraft and passengers, and in-company operation service between operation controllers and aircraft. Aircraft (pilots), ground controllers, passengers, and operation controllers are the primary actors of this system.

As for the air traffic control service, ground controllers and aircraft, service providers and receivers, use radio communication channels to exchange messages each other. It is the basic pattern of interaction between them that an aircraft requests permission to make a move and the ground controller gives instructions on the move. Aircraft provide the transportation service to passengers using aircraft as service media, with which physical transportation is provided. Since assessment of passenger satisfaction was beyond the scope of this study, we did not explicitly model the transportation service and passengers. Operation controllers of an airline provide the operation service to aircraft. They also use radio as the physical communication channels. An operation controller determines the timing of departure request considering the flight schedule and the status of aircraft. The pilot of a departing aircraft requests a departure clearance to the ground controller with an instruction from the operation controller. The operation controller carries out his/her tasks following some scheduling policy of the airline.

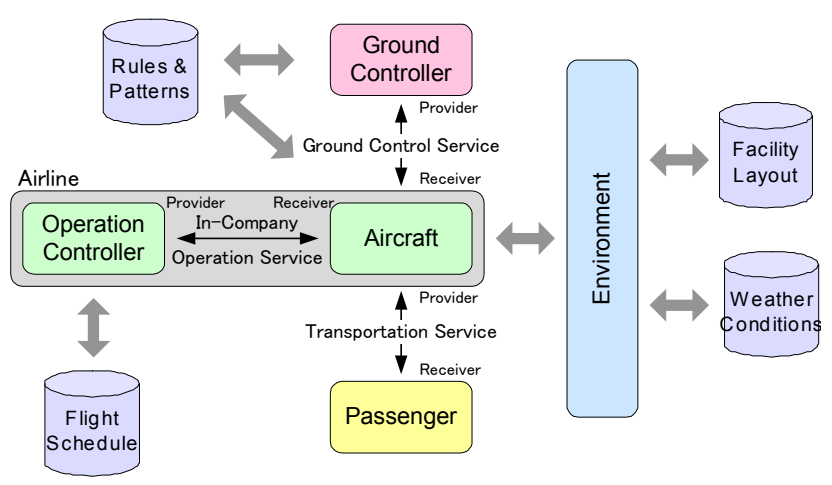

Figure 1. Overview of the simulation model.

\subsection{Agent-Based Approach of Modeling}

An agent-based approach [10] has been adopted for modeling the target service system. An agent here stands for an autonomous 
entity that can determine its own behavior in response to changing environment. An agent-based approach is a method to study a complex system by modeling the system as a collection of many agents. Components that appear in the aircraft operation service were represented as agents of various types, and it is a natural representation of the system. This approach is preferable for model development by prototyping, because each agent can be developed and tested incrementally.

Agents to be modeled in the application domain of this study include aircraft (pilots), ground controllers, operation controllers, passengers, and environment. Each agent takes one of the internal states defined for the agent class. Action rules of an agent can be modularized with such internal states. Action rules and state transition rules were obtained from field observation data and implemented into the agent models.

\subsection{Aircraft Model}

Nine states are defined for an aircraft agent: landing, arrival, stay, towing, pushback, stay after pushback, departure, lineup, and takeoff.

An arriving aircraft is generated as an aircraft agent at the end of runway in the state of "landing." The arriving aircraft agent requests taxi to the ground controller agent after exit from the runway, changes its state to "arrival," and then moves along the taxiing route toward the spot of destination. When the aircraft agent stops at the final destination, it changes its state to "stay."

A staying aircraft agent that has received any instruction from the operation controller agent requests a clearance to the ground controller agent and changes its state. If a staying aircraft agent receives a towing instruction, it is towed in the state of "towing" to the spot of destination and stays there again. There are no distinction of the staying status after taxiing and towing.

If the operation controller agent gives a pushback instruction, a staying aircraft agent changes its state to "pushback" and it is pushed back following the pushback pattern instructed by the ground controller agent. Having finished pushback operation, the aircraft agent changes its state to "stay after pushback." After separation of the towing car and start-up of the engines, the aircraft agent requests a taxi clearance to the ground controller agent.

A departing aircraft agent that has instructed the taxiing route from the ground controller agent changes its state to "departure," and starts to move along the taxiing route. When the aircraft agent arrives at the runway, it requests allowance to enter the runway to the ground controller agent, and it lines up and waits for a takeoff clearance in "lineup." Having obtained the clearance, the aircraft agent changes its state to "takeoff" and starts a takeoff action. When the aircraft agent in takeoff reaches the system boundary, it is eliminated from the system model.

An aircraft agent holds state variables like the current position, moving direction, and velocity in addition to static parameters like its hardware specs. While moving on taxiways, an aircraft agent moves toward the preliminary destination point along the taxiing route, and it moves further to the next destination point if it has passed the previous one. Such behavior to follow consecutive points along the route is consistent with the actual movement of aircraft in taxiing.
The steering and velocity control model determines movement of an aircraft agent. An aircraft agent takes obstacle avoidance behavior and slows down if another aircraft agent enters the area of pilot's cognition, which is defined for each aircraft agent. The velocity control model used in this work is basically equivalent to the optimal velocity model [11], where speed is determined as a function of the distance to the preceding aircraft. This model has been proposed as a microscopic velocity control model of vehicles in traffic congestion. Simulation of such local interactions among aircraft agents enables detailed simulation of aircraft movements and precise evaluation of operation performance.

\subsection{Ground Controller Model}

Seven states are defined for the agent model of ground controller: standby, arrival, pushback, departure, towing, approach, and takeoff. Shifting among these states, the ground controller agent instructs many aircraft agents to move on the ground without any interference. Actually more than one ground controllers instruct aircrafts by dividing areas of the airport surface, but just one ground controller agent instructs all aircrafts. The state of controller model is defined for each aircraft under controller's instruction.

The ground controller agent has no specific tasks in "standby" but monitoring the status of aircraft agents and the ground of airport. It is continuously scanning the current locations of aircraft agents and predicting their movements. If the controller agent receives a request for taxi from an arriving aircraft agent, it plans the taxiing route toward the arriving spot and gives necessary instructions in "arrival." When an aircraft agent ready to depart requests a pushback clearance, the ground controller agent determines the pushback pattern to be used and gives a pushback instruction in "pushback." With a taxi clearance request for departure, the controller agent plans the taxiing route to the runway and gives instructions on the route in "departure." The controller agent that has received a towing request plans the towing route and gives instructions on the route in "towing." The controller agent gives permission to enter the runway in "approach," and then gives a takeoff clearance to an aircraft agent awaiting takeoff.

The architecture of ground controller agent is shown in Figure 2. The ground controller agent performs its tasks in three steps: recognition of the environment, prediction of aircraft movements, and decision-making for instructions. The controller agent recognizes the present situation referring to the information collected from agents representing the external environment: status of the ground, locations of aircraft agents, weather conditions, messages from aircraft agents, and so on. It then predicts movements of aircraft agents in the near future, makes a decision by the internal reasoning mechanism, and then gives instructions to aircraft agents. The reasoning mechanism is based on a heuristic $A^{*}$ search algorithm and a conventional rule-base system that uses the action rules extracted through field observation.

\subsection{Operation Controller Model}

The operation controller agent gives instructions to aircraft agents referring to the flight schedule and considering the present status of aircraft agents. Since it gives instructions from a standpoint of the airline company rather than flight regulation by the authority, two types of instructions are possible: departure and towing instruction. The operation controller agent decides the start timing 
of move with a simple rule to follow the flight schedule in the present system model.

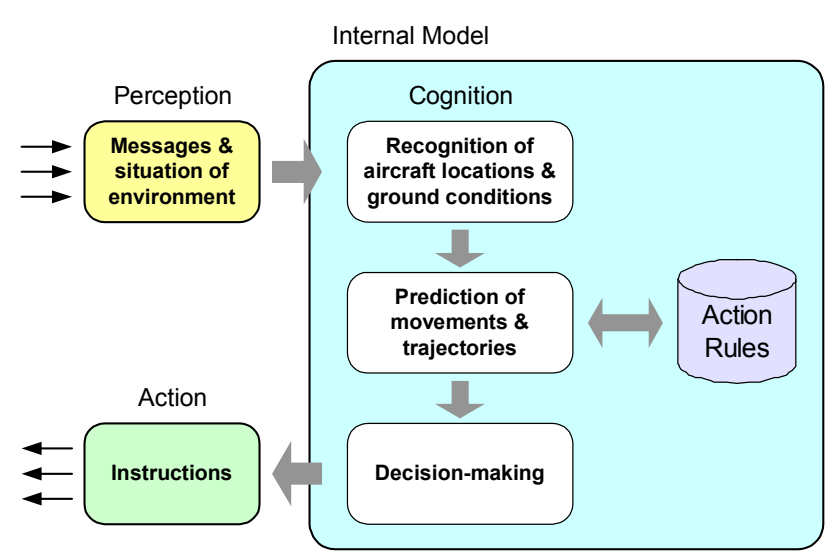

Figure 2. Architecture of ground controller agent.

\subsection{Environment Model}

The environment is modeled also agents for keeping consistency of modeling architecture; it is just an implementation matter. The environment model consists of the ground model and the weather model. It represents the field and the surroundings where airport service is provided. The ground model represents runways, taxiways, and spots. A taxiway is a path where aircraft move between runways and spots. Each taxiway consists of many path segments and joints connecting path segments. A spot is a location where an aircraft parks for embarkation, disembarkation, or maintenance. Wind direction is the only component of the weather model at present. It affects usage of runways, and the ground controller agent decides how to use runways depending on the current status of wind direction. Two patterns are now in use at Tokyo International Airport: one for a north wind and the other for a south wind.

\subsection{Operation Process Model}

Having designed relevant agents for ground aircraft operations, the protocol and process of communication were determined. Aircraft agents carry out the processes by radio communication with the ground controller and operation controller agent. It is the basic style of the air traffic control service that aircraft agents request service to the ground controller agent, and the ground controller agent delivers instructions in reply. In addition, the ground controller agent may spontaneously gives instructions judging from the situation of aircraft agents on airfields. Figure 3 shows the flow of these service processes.

\section{SYSTEM DEVELOPMENT \\ 3.1 Overview of Simulation System}

A simulation system was developed based on the simulation model presented so far. The modules in the simulator could be developed, added, and modified separately due to the flexibility of the multi-agent architecture. Messages between agents are exchanged in a similar manner as verbal communication in the actual work situation. The simulator consists of the component models shown in Figure 1.

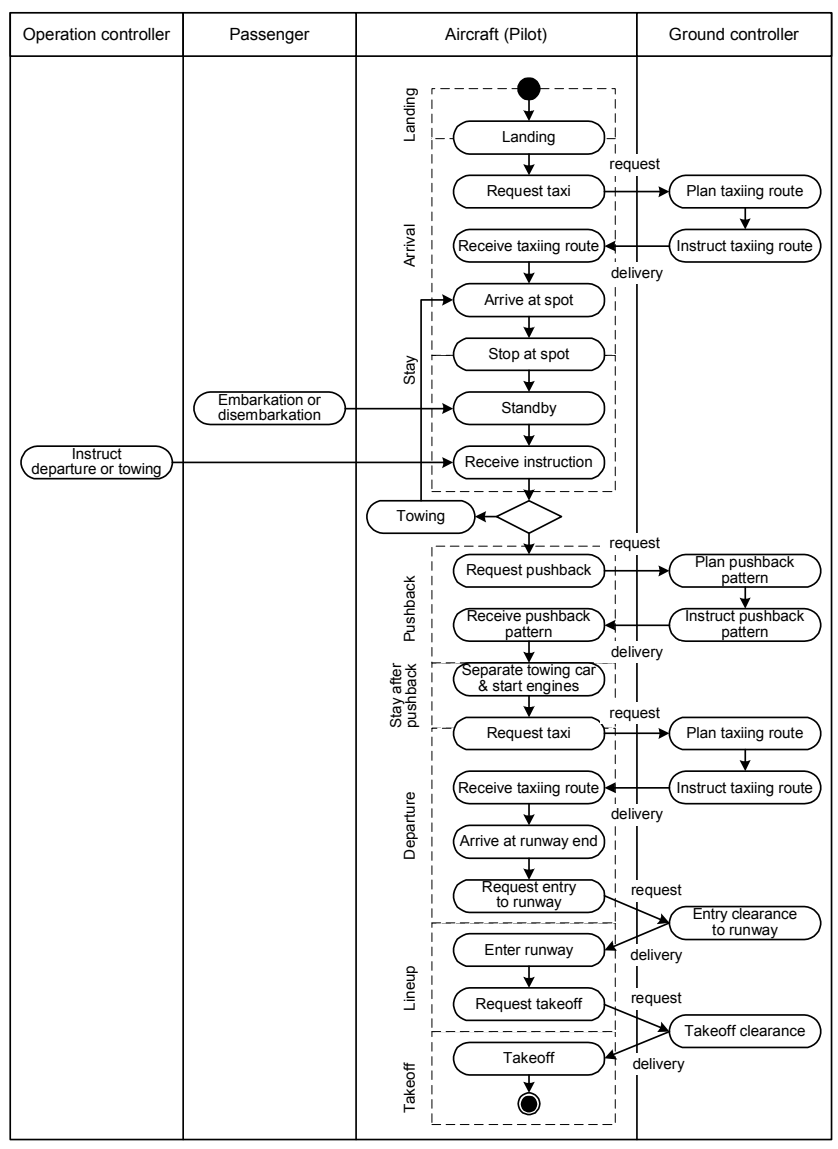

Figure 3. Flow of service processes.

The simulator accepts input data that specifies the simulation scenario such as the layout of airport facilities, flight schedule, weather conditions, and some of the action rules of agents. Outputs of simulation are chronological actions of agents. Results of simulation are visualized using a graphical user interface as shown in Figure 4. Movements of aircraft are displayed by animation of aircraft icons on the airport map in the main window. Flight information like aircraft type, call sign, agent's internal state, destination, and so on are shown in text near the icon. The area of pilot's cognition and the planned taxiing route of each aircraft are also displayed graphically. Messages exchanged between agents are transcribed in the separate communication window.

The visualization of simulation results is helpful not only for the designers but also for the field experts to understand the results intuitively. The field experts who looked at preliminary results of simulation could point out unrealistic movements of aircraft, and it contributed to improve the simulation model through feedback of their opinions. They could also point out problems in the present operation scheme and the causes by looking at visualized simulation results. Visually displaying simulation results thereby is an essential function of the simulation system. 


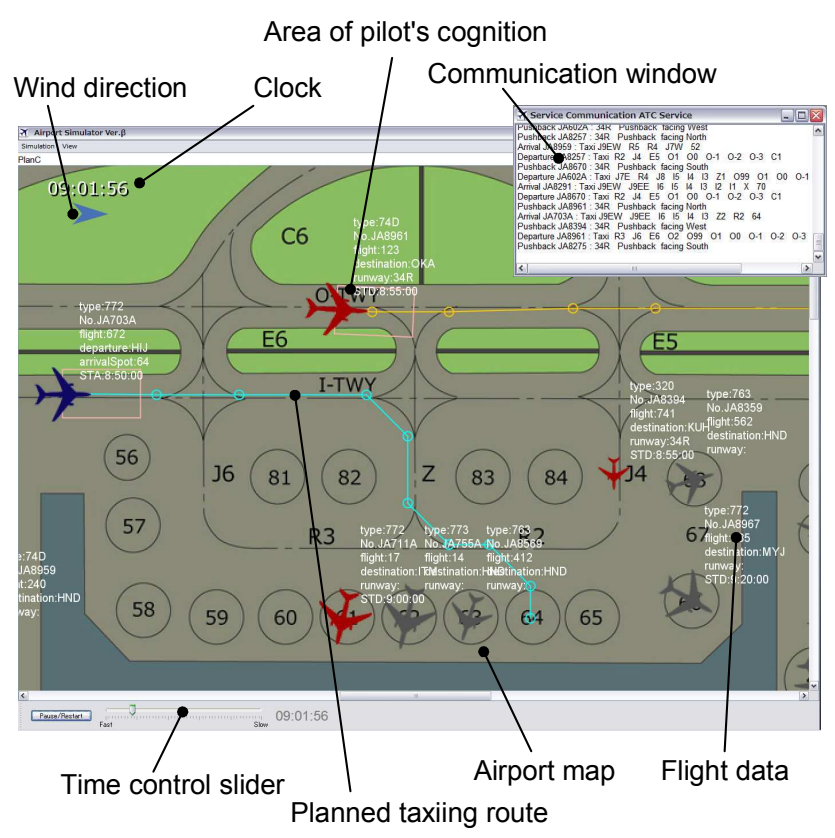

Figure 4. Graphical user interface of simulator.

\subsection{Test Simulation for Model Evaluation}

A test simulation was performed to check the validity of the simulation model. The time required for arrival and that for departure were chosen as performance indicators, because they determine whether or not flights are operated on time and then most affect passengers' convenience in airline business. Fuel savings from reduction of delays contributes to cuts in $\mathrm{CO} 2$ emission as well as operation costs. The time required from pushback request to entrance to the runway for departure, and that from exit from the runway to stop at the spot for arrival were evaluated from the video taken at the airport for three hours during daytime, December 10, 2007. The simulation scenario used for the test was set up from the flight schedule and the weather conditions during the same time period.

The time predicted by simulation was compared with that evaluated from the video. As mentioned before, the simulation model had been improved to eliminate unrealistic behavior of agents by showing preliminary results to the field experts and getting their comments until the final results were obtained. Figure 5 shows that the correlation coefficient is 0.88 and the regression coefficient is 1.016 between simulated and measured data; the simulation could well predict the time required for arrival and departure.

\section{APPLICATION TO FACILITY EXTENSION AT HANEDA}

Tokyo International Airport (Haneda) is the fourth busiest airport in the world for number of passengers over 60 million per year, and more than one third of the domestic passengers in Japan use it. In response to increasing air traffic demands, extension of runways and terminal buildings has been repeated at Haneda. The fourth runway (Runway D) is now under construction expecting its open in 2010, and another terminal building for international flights is planned also. As a result of the new constructions, capacity of the airport is expected to attain 407,000 operations per year, which is 1.3 times larger than the present. Delays during taxiing, however, are getting longer recently despite the past extensions, and whether or not the new goal will be achieved depends on efficient ground aircraft operations after the constructions.

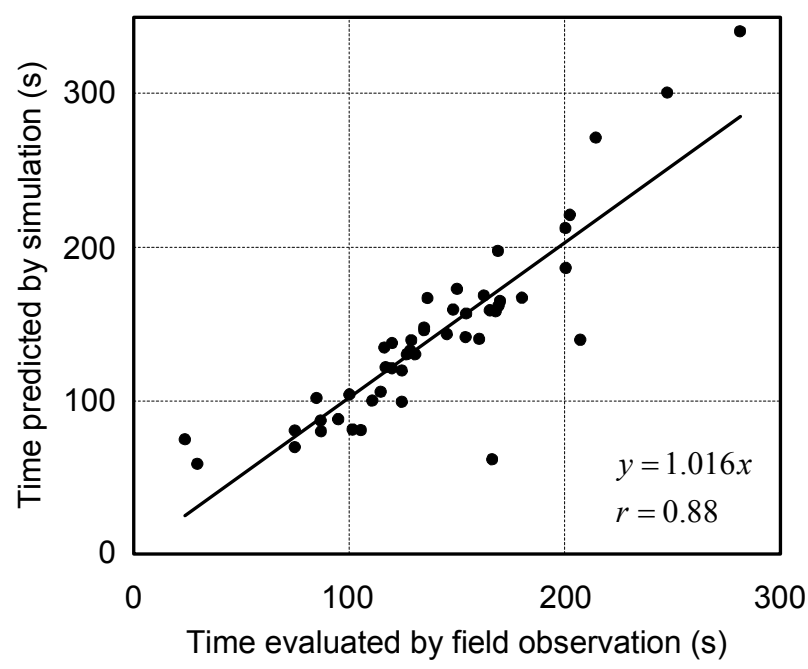

Figure 5. Comparison of time required for arrival and departure.

Ground aircraft operations after the scheduled extension of facilities at Tokyo International Airport were simulated using the simulation system developed in this study to demonstrate usefulness of the approach. Figure 6 shows an overview of the operation scheme for a south wind preliminarily planned after the extension. There is concern with this preliminary plan is that crossing across runways and counter flow of aircraft, which occur at the spots indicated with red circles in Figure 6, will cause stagnation in aircraft flow on airfields and cause delays. Whether or not this concern is realistic was checked by the simulation developed in this study, and the degree of stagnation was evaluated. From the results obtained, modification of operation scheme is under consideration to resolve the problem. The simulation is useful also for this process of redesigning the operation scheme.

\section{CONCLUSION}

A simulation model was constructed for ground aircraft operations at a large airport to realize efficient operations. A multi-agent simulation system was developed based on the proposed model. An ethnographic approach was adopted where expert knowledge and work patterns used by the field staff were extracted through field observation and implemented into the model. An interactive process that simulation results were visualized and shown to the field experts repetitively was very effective to consider feedbacks from the experts in the course of development. 


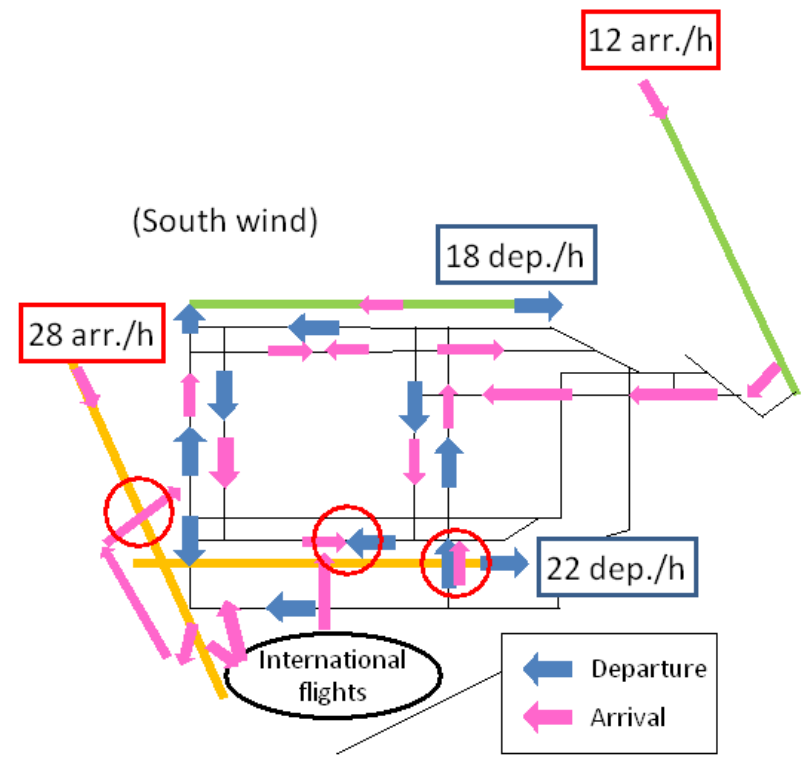

Figure 6. Operation scheme planned after the extension.

The simulation model was evaluated by comparison of simulation results with the field data obtained at Tokyo International Airport. For demonstration purpose, the method was also applied to ground aircraft operations at the same airport after the scheduled extension of a runway and a terminal building. It was demonstrated that the simulation could reveal some problems in the planned operation scheme and how predicted operation performance deviates from the expectation. These findings will be useful to reconsider the present scheme to achieve more efficient aircraft operations.

\section{REFERENCES}

[1] Kazda, A., and Caves, R. E. 2007. Airport Design and Operation. Elsevier, Oxford, UK.

[2] Zografos, K. G., and Madas, M. A. 2007. Advanced Modeling Capabilities for Airport Performance Assessment and Capacity Management. J. of Transportation Research Board, 2007, Transportation Research Board of the National Academies, Washington, D.C., 60-69.

[3] Baik, H., Sherali, H. D., and Trani, A. A. 2002. TimeDependent Network Assignment Strategy for Taxiway Routing at Airports. J. of the Transportation Research Board, 1788, Transportation Research Board of the National Academies, Washington, D.C., 70-75.

[4] Martinez, J. C., Trani, A. A., and Ioannou, P. G. 2001. Modeling Airside Airport Operations Using General-Purpose, Activity-Based, Discrete-Event Simulation Tools. J. of the Transportation Research Board, 1744, Transportation Research Board of the National Academies, Washington, D.C., 65-71.

[5] Simmod Manual - How Simmod Works. US Federal Aviation Administration. http://www.tc.faa.gov/acb300/ how_simmod_works.pd. Accessed on October 7, 2009.

[6] MIDAS: Man-Machine Integration Design and Analysis System. NASA. humansystems.arc.nasa.gov/groups/ midas/index.html. Accessed on July 15, 2009.

[7] Chiu, C. Y., and Walton, C. M. 2002. Integrated Simulation Method to Evaluate the Impact of New Large Aircraft on Passenger Flows at Airport Terminals. J. of the Transportation Research Board, 1788, Transportation Research Board of the National Academies, Washington, D.C., 83-92.

[8] Suchman, L. 1987. Plans and Situated Actions. Cambridge University Press, Cambridge, UK.

[9] Buur, J., Binder, T., and Brandt, E. 2000. Taking Video beyond 'Hard Data' in User Centered Design. Participatory Design Conference, 121-131.

[10] Ferber, J. 1999. Multi-Agent Systems: An Introduction to Distributed Artificial Intelligence. Addison Wesley, Harlow, UK.

[11] Bando, M., Hasebe, K., Nakayama, A., Shibata, A., and Sugiyama, Y. 1995. Dynamic model of traffic congestion and numerical simulation. Physical Review, E51, 1035-1042. 